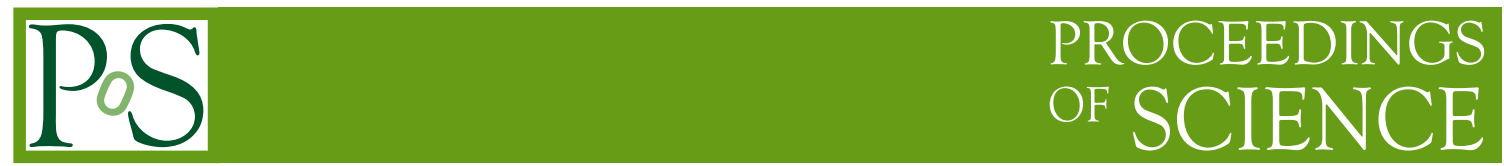

\title{
Open charm physics with heavy ions: experimental results
}

\section{André Mischke*}

Institute for Subatomic Physics, Department for Physics and Astronomy and EMME $\phi$, Faculty of Science, Utrecht University, Princetonplein 1, 3584 CS Utrecht, the Netherlands

E-mail: a.mischkeduu.nl

This contribution gives an overview of current experimental results on open charm production in high-energy collisions of atomic nuclei, presented at the $8^{\text {th }}$ edition of the International Workshop On Charm Physics. Open charm was studied in elementary proton-proton interactions as well as in proton-lead and lead-lead collisions at the Large Hadron Collider (LHC).

VIII International Workshop On Charm Physics 5-9 September, 2016

Bologna, Italy

\footnotetext{
* Speaker.
} 


\section{Introduction}

Quantum Chromodynamics (QCD) is the fundamental theory describing the strong interaction between quarks and gluons. One of the characteristic features of QCD is asymptotic freedom where the interaction becomes weak at large momentum transfer. In contrast, the potential between the quarks rises steeply when the partons are separated. In fact, the binding force becomes so strong that under "normal conditions" quarks and gluons are permanently confined inside hadrons. A fascinating and direct consequence of asymptotic freedom is that under the conditions of sufficiently high temperature or baryon density the strongly interacting quarks and gluons are liberated from their hadronic confinement. This new state of matter is called the Quark-Gluon Plasma (QGP), in which the basic degrees of freedom are released. The properties of such matter are fundamental predictions of QCD and its study is one of the leading and most active fields in contemporary subatomic physics. The goal of ultra-relativistic heavy-ion physics is to create this plasma in the laboratory and carefully study its properties [1,2].

Quarks and gluons (partons) of the incoming nuclei can undergo hard collisions in the early stage of the collisions. In elementary proton-proton interactions these hard-scattered partons fragment into jets of collimated, high transverse momentum particles. In ultra-relativistic heavy-ion collisions on the other hand, these very energetic partons traverse through the also formed plasma and get slowed down through medium-induced gluon radiation. The properties of the QGP can be studied by the attenuation of these energetic particles [3].

To get a better understanding on the parton energy-loss mechanisms it is important to study its dependance on the colour charge and mass [4] that allows to get a profound understanding on the dynamical properties of the QGP. Heavy quarks (charm and beauty) are ideal probes to perform these studies. Charm quarks are about 250 times heavier than the light up and down quarks that dominate the QGP phase, beauty quarks are 3-4 heavier than charm quarks, and their masses do not change by chiral symmetry breaking [5]. Due to their large mass, heavy quarks are predominantly produced in the initial hard partonic scattering in the early stages of the collision via gluon fusion processes [6] and therefore probe the complete space-time evolution of the plasma phase. As previous measurements have shown [7, 8], heavy-quark production by initial state gluon fusion also dominates in heavy-ion collisions where many in part overlapping nucleon-nucleon collisions occur. Thermal production later in the collision might have a small contribution to the heavy-quark production at low transverse momentum [9]. In addition to the parton energy loss, heavy quarks are expected to also experience collisions with the medium constituents (collisional energy loss).

The data reported in this contribution were obtained with the ALICE, ATLAS, CMS and LHCb experiment at the CERN-LHC [10]. I will concentrate on the discussion of the D-meson measurements in elementary proton-proton interactions as well as in proton-lead and lead-lead collisions. The different D-meson species are reconstructed in the hadronic decay channel using secondary vertexing. A detailed description of the measurements and analysis techniques are given in [11].

\section{2. pp system: QCD vacuum}

Open charm production measurements in proton-proton interactions provide crucial tests of predictions from perturbative QCD (pQCD) models and serve as an important baseline for the mea- 

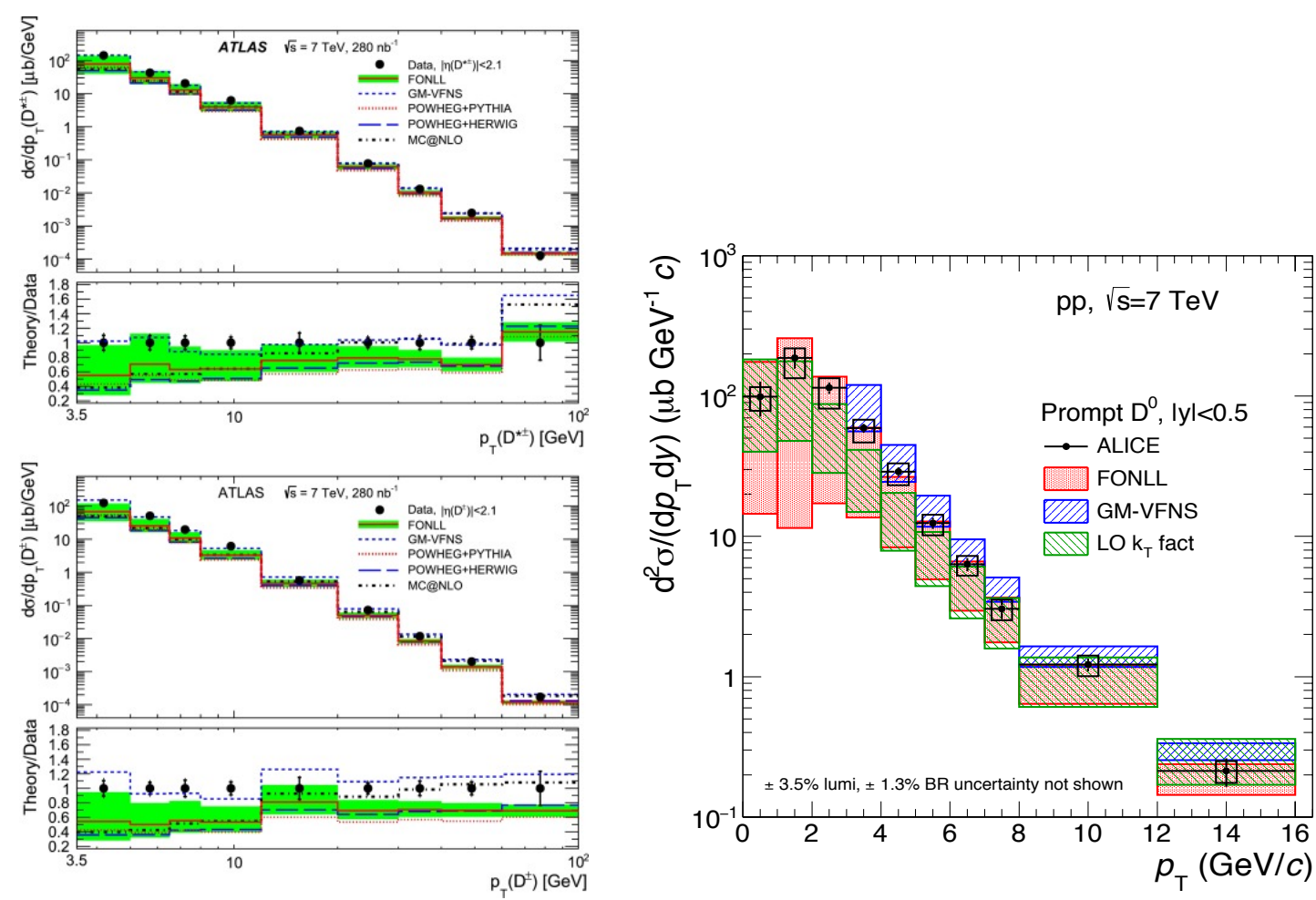

Figure 1: Invariant production cross section of $\mathrm{D}^{\star \pm}$ - and $\mathrm{D}^{ \pm}$-mesons $[12,13]$ (left panel) and $\mathrm{D}^{0}$-meson at mid rapidity $[14,15]$ (right panel) in proton-proton collisions at $\sqrt{s}=7 \mathrm{TeV}$. The data are compared with next-to-leading-order (FONLL, GM-VFNS, POWHEG and MC@NLO) and leading order $k_{\mathrm{T}}$-factorisation perturbative QCD calculations.

surements in heavy-ion collisions.

The invariant open charm production cross section was measured in inelastic pp collisions at $\sqrt{s}=$ 2.76, 7 and $13 \mathrm{TeV}$. As an example, Fig. 1 depicts the production cross at mid rapidity for $\mathrm{D}^{\star \pm}$ - and $\mathrm{D}^{ \pm}$-mesons [12,13] (left panel) and $\mathrm{D}^{0}$-mesons $[14,15]$ (right panel) in proton-proton collisions at $\sqrt{s}=7 \mathrm{TeV}$. Remarkable is the ALICE measurement down to $p_{\mathrm{T}}=0$. The LHCb Collaboration provided similar measurement at forward rapidities $2.0<y<4.5[16,17]$. The data in Fig. 1 are compared with state-of-the-art next-to-leading-order (NLO), such as FONLL, GM-VFNS, POWHEG and MC@NLO, and leading order $k_{\mathrm{T}}$-factorisation pQCD calculations. The data are systematically higher than the central value of the calculations, but consistent within the relatively large theoretical uncertainties. Please note that the measured beauty production cross section agrees with NLO pQCD calculations in the studied $\sqrt{s}$ range [18].

The ATLAS Collaboration has measured the $\mathrm{D}^{\star \pm}$-meson production in jets in pp collisions at $\sqrt{s}=7 \mathrm{TeV}$ for jets with a transverse momentum between 25 and $70 \mathrm{GeV}$ [19]. Figure 2 shows the ' $\mathrm{D}^{\star \pm}$ in jet' production rate $(R)$ at mid-rapidity $|\eta|<2.5$ for two different jet energies. The data are compared with NLO pQCD calculations using the Monte Carlo event generators PYTHIA and HERWIG for parton showering. The predicted values of production rate by PYTHIA and POWHEG+PYTHIA are very similar, which is also the case when comparing calculations from HERWIG and POWHEG+HERWIG, as expected. Since $R$ is defined as the ratio between the number of $\mathrm{D}^{\star \pm}$ 

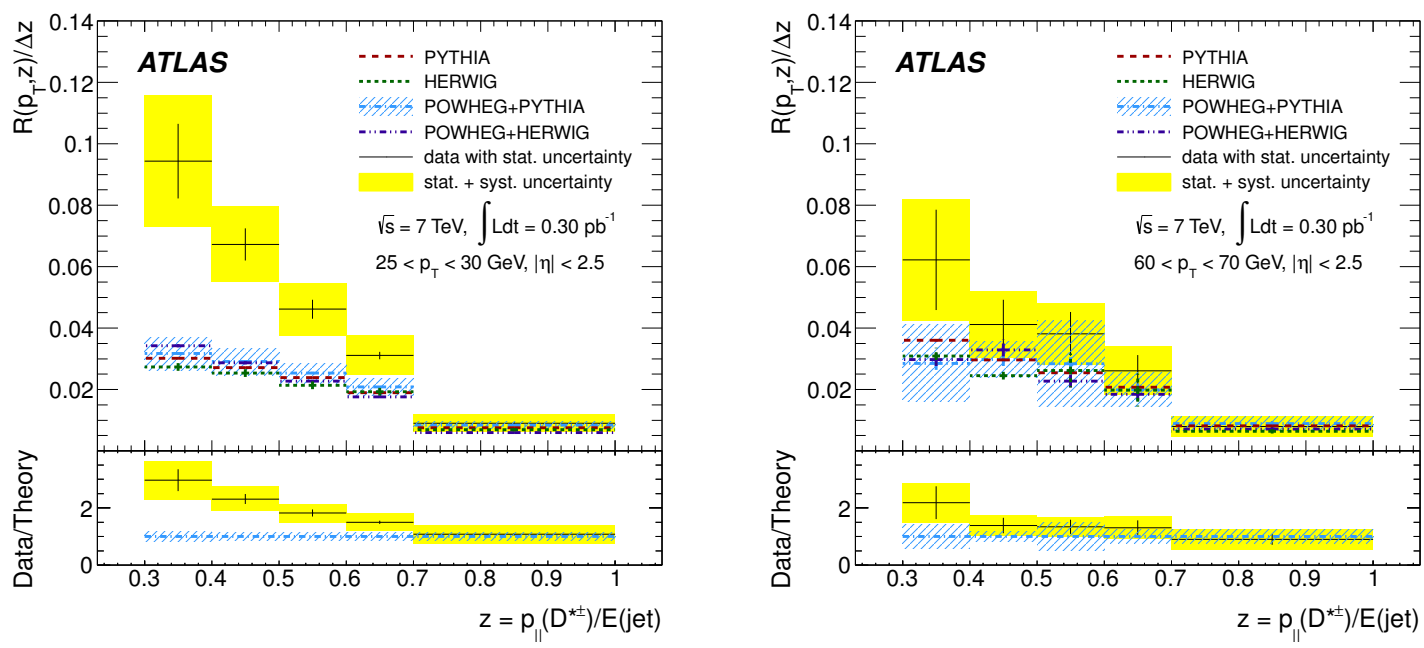

Figure 2: $\mathrm{D}^{\star \pm}$-meson production rate $R\left(p_{\mathrm{T}}, z\right) / \Delta z$ in the jet $p_{\mathrm{T}}$ range $25-30 \mathrm{GeV}$ (left panel) and $60-70 \mathrm{GeV}$ (right panel) versus $z$, measured in $7 \mathrm{TeV}$ proton-proton interactions [19] and compared with predictions from two different Monte Carlo event generators.

jets and inclusive jets, the changes of total jet cross sections and $p_{\mathrm{T}}$ distributions between LO and NLO QCD calculations largely cancel. However, the Monte Carlo calculations fail to describe the data at small fractional momentum of the $\mathrm{D}^{\star \pm}$ mesons $(z)$. This discrepancy is strongest at low jet transverse momentum and cannot be explained by varying the mixture of charm and beauty jets in the Monte Carlo calculations. This is an indication that jet fragmentation into $\mathrm{D}^{\star \pm}$ mesons is not well modelled in current Monte Carlo generators.

\section{A-A system: QGP formation (hot and dense QCD medium)}

Heavy quarks (charm and beauty) are ideal probes to study the properties of the QGP that is created in nucleus-nucleus collisions since they experience the full space-time evolution of the expanding system. They propagate through the medium and lose energy due to gluon radiation and multiple collisions.

The radiative parton energy loss depends on the medium properties such as the density, temperature and mean free path, which is usually quantified in the so-called transport coefficient $\hat{q}$. Moreover, it depends on the path length in the medium and the parton properties such as the colour charge and the mass [20]. Theoretical models [21,22] predicted that heavy quarks should experience a smaller energy loss than light quarks while propagating through the plasma due to the suppression of smallangle gluon radiation, the so-called dead-cone effect. Nuclear effects are typically quantified using the nuclear modification factor $R_{\mathrm{AA}}$ where the particle yield in nucleus-nucleus collisions is divided by the yield in pp interactions, scaled by the averaged number of binary collisions. The latter is obtained from Glauber calculations. An $R_{\mathrm{AA}}$ of one would indicate that no nuclear effects, neither "cold" (such as Cronin, shadowing or gluon saturation) nor "hot" (parton energy loss) are present and that nucleus-nucleus collisions can be considered as an incoherent superposition of nucleonnucleon interactions. The comparison of the nuclear modification factor of charged pions $\left(R_{\mathrm{AA}}^{\pi^{ \pm}}\right)$, mostly originating from gluon fragmentation at the TeV-energy scale, with that of charm $R_{\mathrm{AA}}^{\mathrm{D}}$ and 


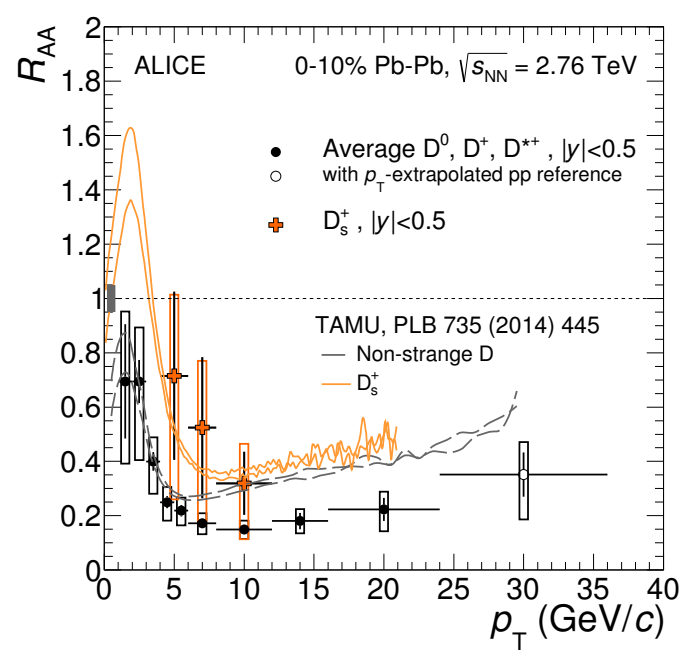

Figure 3: Nuclear modification factor $R_{\mathrm{AA}}$ of prompt, average $\mathrm{D}$ [23] and $\mathrm{D}_{\mathrm{s}}^{+}$mesons [24] at mid-rapidity in the $10 \%$ most central lead-lead collisions at $\sqrt{s_{\mathrm{NN}}}=2.76 \mathrm{TeV}$ compared with the TAMU energy loss model [25].

beauty hadrons $R_{\mathrm{AA}}^{\mathrm{B}}$ allow the study of the parton colour charge and mass dependence of the energy loss.

Figure 3 illustrates the nuclear modification factor for prompt D mesons at mid-rapidity in lead-lead collisions at $\sqrt{s_{\mathrm{NN}}}=2.76 \mathrm{TeV}$ [23]. The D-meson yield for the most central events is strongly suppressed (by factor of $\approx 5$ at around $10 \mathrm{GeV} / \mathrm{c}$ ). Energy-loss models currently describe the observed suppression at high transverse momentum reasonably well.

Furthermore, the ALICE experiment studied the $R_{\mathrm{AA}}$ of $\mathrm{D}_{\mathrm{s}}^{+}$mesons [24] in nucleus-nucleus collisions (cf. Fig. 3). The $\mathrm{D}_{\mathrm{s}}^{+}$meson is of particular interest since it is a charm-anti-strange quark state. Earlier measurements have shown [1] that strangeness production is enhanced in nucleus-nucleus
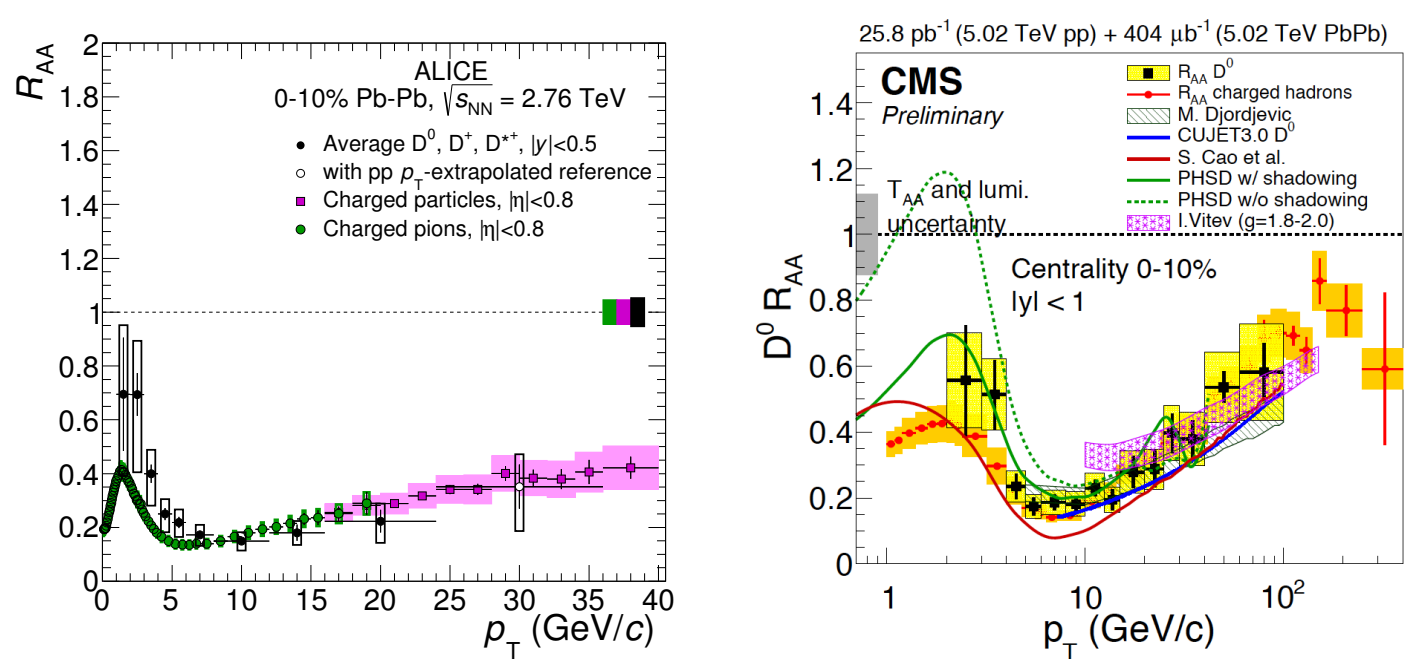

Figure 4: Prompt D-meson and charged hadron $R_{\mathrm{AA}}$ at mid-rapidity in the $10 \%$ most central $\mathrm{Pb}-\mathrm{Pb}$ collisions at $\sqrt{s_{\mathrm{NN}}}=2.76 \mathrm{TeV}$ [23] (left panel) and 5.02 TeV (right panel) [26]. Different model calculations are compared with the $5.02 \mathrm{TeV}$ data. 


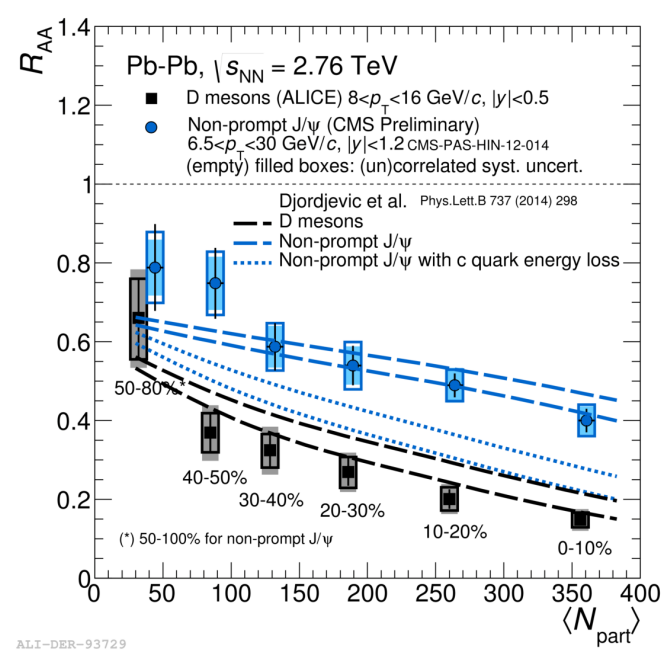

Figure 5: $R_{\mathrm{AA}}$ of prompt D-mesons [27] and J/ $\Psi$ from beauty decays [28] versus the number of participating nucleons.

collisions, so one would expect that the strange D-meson yield at intermediate transverse momentum should be enhanced if charm hadronises via recombination in the medium. Model calculations show [25] that the difference between the $\mathrm{D}$ - and $\mathrm{D}_{\mathrm{s}}^{+}$-meson $R_{\mathrm{AA}}$ should be small (cf. Fig. 3). The ALICE data do not indicate a difference within the current statistical and systematic uncertainties. The prompt D-meson $R_{\mathrm{AA}}$ was measured in $\mathrm{Pb}-\mathrm{Pb}$ collisions at $\sqrt{s_{\mathrm{NN}}}=2.76 \mathrm{TeV}$ (ALICE experiment [23]) and $5.02 \mathrm{TeV}$ (CMS experiment [26]), as shown in Fig. 4. Remarkably, the CMS measurement of the D-meson $R_{\mathrm{AA}}$ goes up to $100 \mathrm{GeV} / c$. The D-meson yields at $5<p_{\mathrm{T}}<10$ $\mathrm{GeV} / c$ are suppressed to the same level as observed for light-quark hadrons [24]. The increasing trend of the yield at high transverse momentum can be understood in terms of the hardening of the particle spectrum. There is an indication that prompt $\mathrm{D}$ mesons are less suppressed than light-quark hadrons at low transverse momentum but the current statistical and systematic uncertainties does not allow a final interpretation of the data.

The centrality dependence of prompt D-meson $R_{\mathrm{AA}}$ [27] is depicted in Fig. 5 together with the $R_{\mathrm{AA}}$ of $\mathrm{J} / \Psi$ from beauty decays (non-prompt $\mathrm{J} / \Psi$ ) [28]. There is a first indication that beauty is less suppressed than charm in heavy-ion collisions. This is also supported by model calculations including quark-mass dependent energy loss. However, there remains an uncertainty from the comparison in a proper kinematical range. This urge the need for the measurement of fully reconstructed $\mathrm{B}$ mesons in nucleus-nucleus collisions over a broad kinematical range.

\section{4. p-A system: Cold nuclear matter effects}

The quantitative understanding of the heavy-ion data in terms of parton energy loss urges the need to disentangle hot nuclear matter from cold nuclear matter effects. Cold nuclear matter effects from the initial state arise from the nuclear modification of the parton distribution functions (shadowing), gluon saturation from evolution equations (DGLAP and BFKL), $k_{\mathrm{T}}$ broadening and Cronin enhancement from multiple parton scatterings, and initial-state energy loss [29, 30, 31, 32]. Initialstate effects can be investigated in proton-lead collisions. 

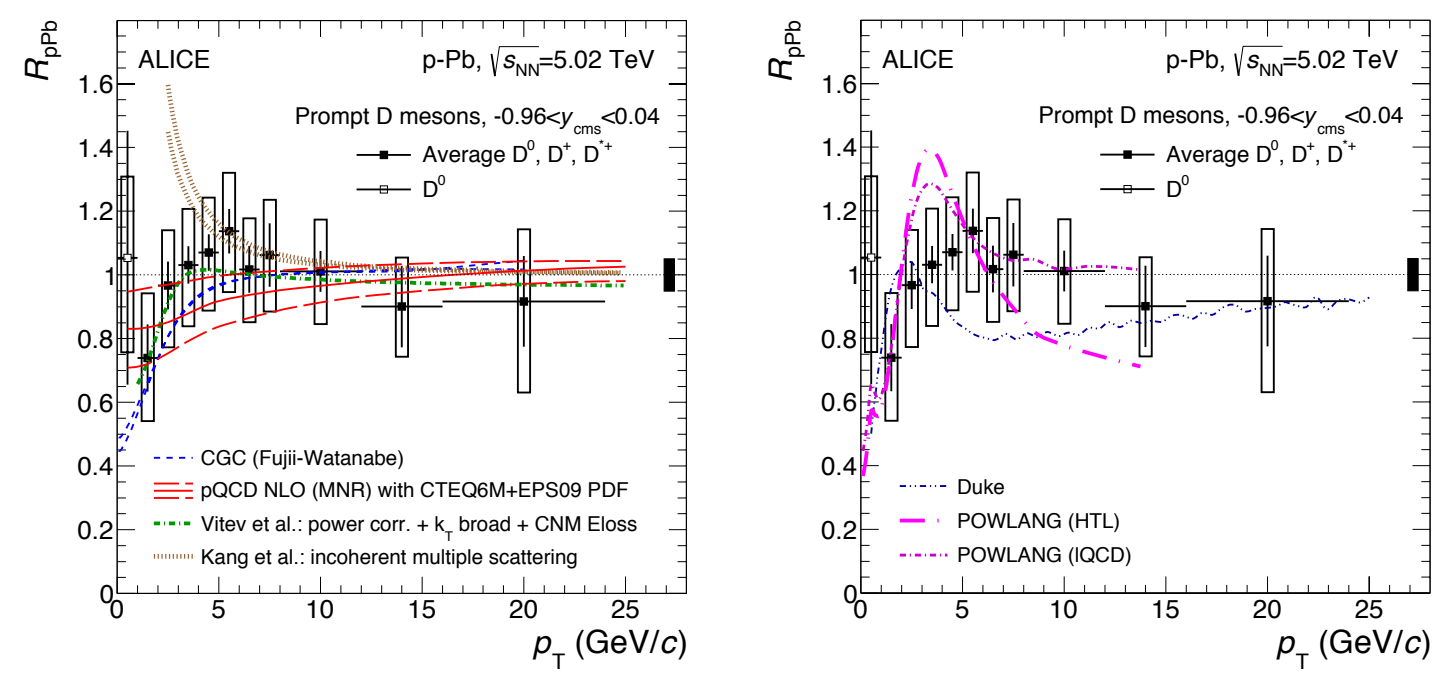

Figure 6: $R_{\mathrm{AA}}$ of the average, prompt $\mathrm{D}$ meson versus $p_{\mathrm{T}}$ in minimum bias proton-lead collisions at $\sqrt{s_{\mathrm{NN}}}=$ $5.02 \mathrm{TeV}$ [33]. The data are compared with models with cold nuclear matter effects only [34, 35] (left panel) and with a "small QGP phase" (right panel).

The nuclear modification factor $R_{\mathrm{pPb}}$ of prompt $\mathrm{D}$ mesons in proton-lead (p- $\mathrm{Pb}$ ) collisions at $\sqrt{s_{\mathrm{NN}}}=5.02 \mathrm{TeV}$ [33] is shown in Fig. 6 and is compatible with unity within systematic uncertainties over the full measured $p_{\mathrm{T}}$ range. Thus, the strong suppression of the D-meson yield observed in central $\mathrm{Pb}-\mathrm{Pb}$ collisions is a final-state effect and arising from the energy loss of the heavy quarks in the plasma. The data are consistent with predictions from shadowing [34] and color glass models [35], which consider cold nuclear matter (CNM) effects only. The data disfavour a suppression larger than $15 \%$ at high $p_{\mathrm{T}}$, which rule out models with a "small plasma phase" (e.g. POWLANG).

The CMS experiment measured the nuclear modification factor $R_{\mathrm{pA}}$ of fully reconstructed $\mathrm{B}$ mesons in the exclusive hadronic decay channel $B^{+} \rightarrow J / \Psi K^{+} \rightarrow \mu^{+} \mu^{-} K^{+}$at mid-rapidity $\left(y_{\text {lab }}<2.4\right)$ and $10<p_{\mathrm{T}}<60 \mathrm{GeV} / c$ in p-Pb collisions at $\sqrt{s_{\mathrm{NN}}}=5.02 \mathrm{TeV}$ [36] (cf. Figure 7) that is unity and shows no $p_{\mathrm{T}}$ dependence. Thus, no indication of significant cold nuclear matter effects on beauty production were found, considering the statistical and systematic uncertainties, when compared to pp FONLL calculations scaled by the number of incoherent nucleon-nucleon collisions. This result provides a baseline for the measurements of the in-medium energy loss of beauty-quarks in lead-lead collisions.

Furthermore, charm [37, 38] and beauty jets [39] were measured in proton-lead collisions at $\sqrt{s_{\mathrm{NN}}}=5.02 \mathrm{TeV}$ by the CMS experiment. Charm jets are identified by requiring a secondary vertex comprised of three or more charged tracks that are significantly displaced from the primary vertex. A variant of the secondary vertex mass is used to extract the relative contributions of jet flavours. Jets from beauty-quark fragmentation are found by exploiting the long lifetime of hadrons containing a $b$ quark through tagging methods using distributions of the secondary vertex 


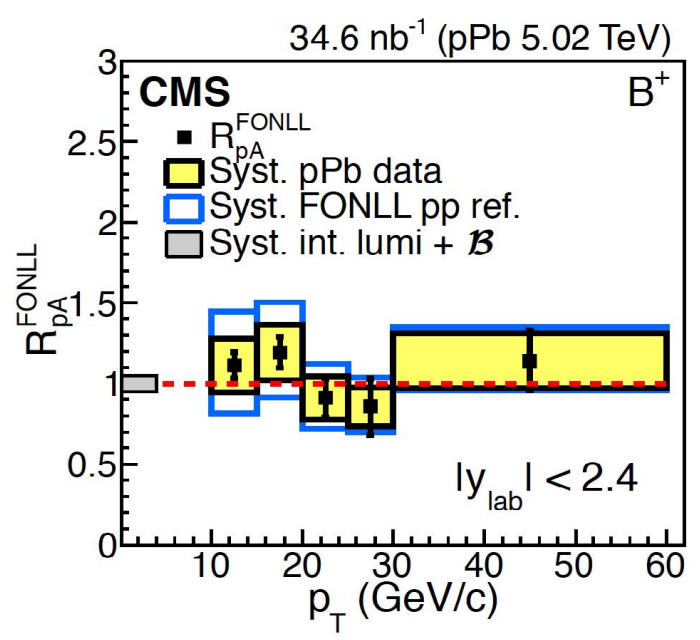

Figure 7: The nuclear modification factor $R_{\mathrm{pA}}^{\mathrm{FONLL}}$ of $B^{+}$mesons in $\mathrm{p}-\mathrm{Pb}$ collisions at $\sqrt{s_{\mathrm{NN}}}=5.02 \mathrm{TeV}$ as a function of $p_{\mathrm{T}}$ [36]. The statistical and systematic uncertainties on the $\mathrm{p}-\mathrm{Pb}$ data are shown as bars and yellow boxes around the data points, respectively. The systematic uncertainties from the FONLL predictions are plotted separately as open blue boxes. The global systematic uncertainties are shown as full grey boxes at unity.

mass and displacement. The extracted cross sections for $\mathrm{b}$ jets are scaled by the effective number of nucleon-nucleon collisions and are compared to a reference obtained from PYTHIA simulations of pp collisions. The charm-jet $p_{\mathrm{T}}$ differential cross section, shown in the left panel of Fig. 8, is consistent with PYTHIA calculations. The inclusive beauty-jet $R_{\mathrm{pPb}}$ is in agreement with the pp reference (cf. right panel of Figure 8). In conclusions, no significant CNM effects on heavy-flavour production at high- $p_{\mathrm{T}}$ is observed.
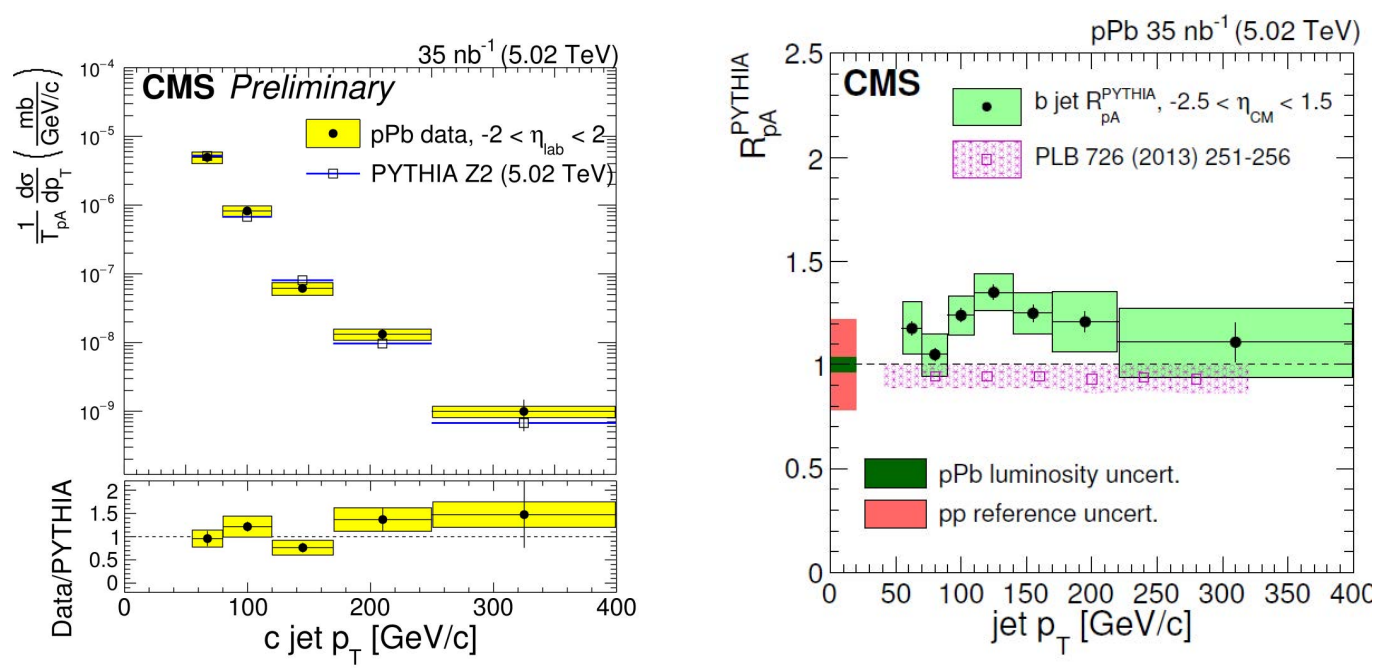

Figure 8: Charm-jet $p_{\mathrm{T}}$ differential cross section compared with PYTHIA calculations [37, 38] (left panel) and inclusive beauty-jet $R_{\mathrm{pPb}}$ versus $p_{\mathrm{T}}$ in $\mathrm{p}-\mathrm{Pb}$ collisions at $\sqrt{s_{\mathrm{NN}}}=5.02 \mathrm{TeV}$ [39] (right panel). 

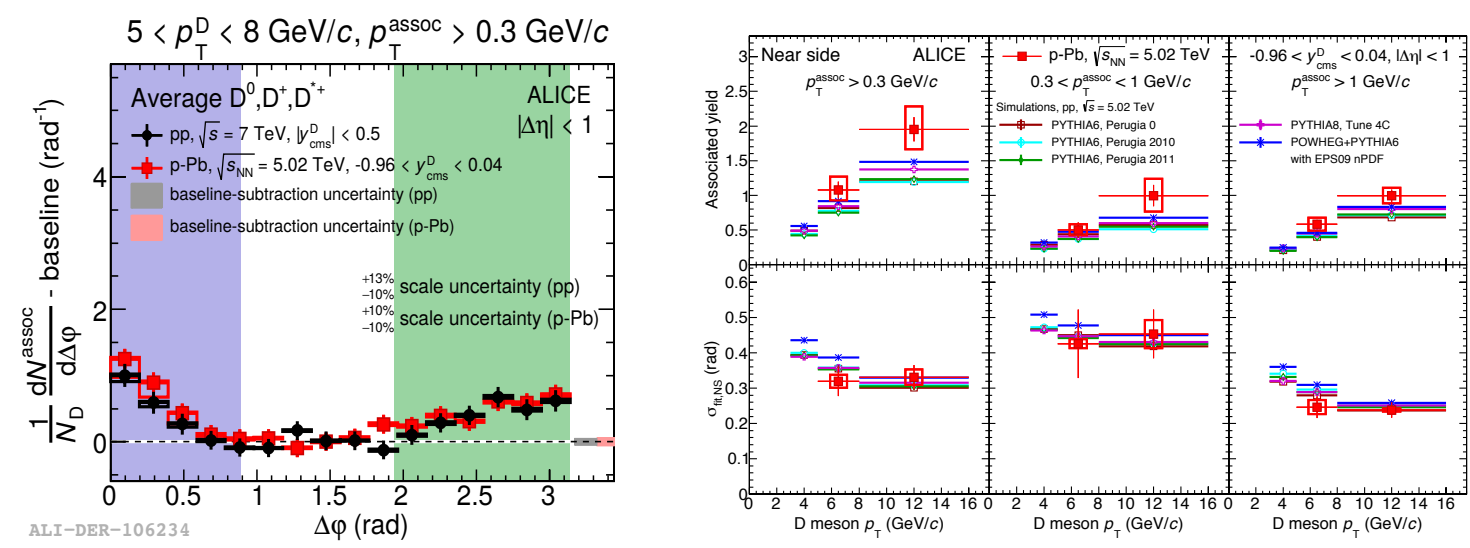

Figure 9: Left panel: Azimuthal correlation distribution of $\mathrm{D}$ mesons with $5<p_{\mathrm{T}}^{\mathrm{D}}<8 \mathrm{GeV} / c$ and and charged particles with $p_{\mathrm{T}}^{\text {assoc }}>0.3 \mathrm{GeV} / c$ at mid-rapidity in pp collisions at $\sqrt{s}=7 \mathrm{TeV}$ and in $\mathrm{p}-\mathrm{Pb}$ collisions at $\sqrt{s_{\mathrm{NN}}}=5.02 \mathrm{TeV}$ after baseline subtraction. Right panel: Near-side correlation associated yield (top row) and near-side peak width (bottom row) measured in $\mathrm{p}-\mathrm{Pb}$ collisions at $\sqrt{s_{\mathrm{NN}}}=5.02 \mathrm{TeV}$ [42] compared with the results from different Monte-Carlo event generators. Statistical and systematic uncertainties are shown as error bars and boxes, respectively.

Correlations between heavy quarks are largely not effected by the fragmentation process in protonproton interactions. In heavy-ion collisions, however, the medium alters the fragmentation process, so that observables are sensitive to the properties of the medium [40, 41]. It has been shown that the fragmentation function, which describes how the parton momentum is distributed among the final state hadrons, is most suited for these detailed studies.

The azimuthal correlations of D mesons and charged particles were measured for the first time with the ALICE detector in pp collisions at $\sqrt{s}=7 \mathrm{TeV}$ and $\mathrm{p}-\mathrm{Pb}$ collisions at $\sqrt{s_{\mathrm{NN}}}=5.02 \mathrm{TeV}$ [42]. The azimuthal angular correlation distribution of D mesons with $5<p_{\mathrm{T}}^{\mathrm{D}}<8 \mathrm{GeV} / c$ and and charged particles with $p_{\mathrm{T}}^{\text {assoc }}>0.3 \mathrm{GeV} / c$ at mid-rapidity in pp collisions at $\sqrt{s}=7 \mathrm{TeV}$ and in $\mathrm{p}-\mathrm{Pb}$ collisions at $\sqrt{s_{\mathrm{NN}}}=5.02 \mathrm{TeV}$ is depicted in Fig. 9 (left panel). The distributions were fitted with two Gaussians after baseline subtraction. The near-side correlation peak is sensitive to characteristics of jets containing a D meson. The right panel of Fig. 9 illustrates the extracted fit parameters (nearside associated yield and peak width). Similar yields were measured for $\mathrm{pp}$ and $\mathrm{p}-\mathrm{Pb}$ collisions and the data are well reproduced by PYTHIA simulations within uncertainties in the studied kinematic ranges.

\section{Summary}

The Large Hadron Collider provided precision data for the measurement of heavy-flavour production in pp collisions at different energies to test predictions from pQCD.

Proton-lead collisions allows the study of cold-nuclear effects such as shadowing and gluon saturation. No significant modification is observed for the production of prompt $\mathrm{D}$ mesons, B mesons, charm and beauty jets at sufficient high $p_{\mathrm{T}}$. The run-2 $\mathrm{p}-\mathrm{Pb}$ data taking at 5 and $8 \mathrm{TeV}$ collision energy in 2016 will allow refining these measurements. 
The large suppression of the $\mathrm{D}$-meson yield in $\mathrm{Pb}-\mathrm{Pb}$ collisions is a final-state effect and arises from interactions of the charm quark with the hot QCD medium. No indications were found for a smaller energy loss of D mesons compared to light-quark hadrons within the experimental uncertainties. The centrality dependence of the $R_{\mathrm{AA}}$ for prompt $\mathrm{D}$ mesons and $J / \Psi$ mesons from $\mathrm{B}$ decays shows a clear indication for a different suppression of the production yields, in agreement with the expected quark-mass dependence of the parton energy loss.

The increase of the interaction rate for the LHC run-3 after the second long shutdown in 2019/20 will require a significant upgrade of the experiments to improve substantially the current performances, especially for the measurements of heavy-flavour particles and jets in nucleus-nucleus collisions.

\section{Acknowledgments}

Sincerest thanks to the ALICE, ATLAS, CMS and LHCb Collaboration for providing the data and the LHC accelerator team. This work was supported by the Netherlands Organisation for Scientific Research (project number: 680-47-232) and the Dutch Foundation for Fundamental Research (project numbers: 12PR3083).

\section{References}

[1] N. Brambilla et al., Eur. Phys. J. C 74, 2981 (2014).

[2] B.V. Jacak and B. Müller, Science 337, 310 (2012).

[3] B. Müller, J. Schukraft and B. Wyslouch, Ann. Rev. Nucl. Part. Sci. 62, 361 (2012).

[4] N. Armesto et al., Phys. Rev. D 71, 054027 (2005).

[5] B. Müller, Nucl. Phys. A 750, 84 (2005).

[6] Z. Lin and M. Gyulassy, Phys. Rev. C 51, 2177 (1995).

[7] S.S. Adler et al., Phys. Rev. Lett. 94, 082301 (2005).

[8] Y. Zhang et al., J. Phys. G: Nucl. Part. Phys. 32, S529 (2006).

[9] J. Uphoff, O. Fochler, Z. Xu and C. Greiner, Phys. Rev. C 82, 044906 (2010).

[10] A. Breskin and R. Voss, JINST 3, S08001-S08007 (2008).

[11] A. Andronic et al., Eur. Phys. J. C 76, 107 (2016).

[12] G. Aad et al. (ATLAS Collaboration) Nucl. Phys. B 907, 717 (2016).

[13] D.H. Guest for the ATLAS Collaboration, these proceedings.

[14] B. Abelev et al. (ALICE Collaboration), arXiv:1605.07569 and JHEP 1201, 128 (2012).

[15] A. Grelli for the ALICE Collaboration, these proceedings.

[16] R. Aaij et al. (LHCb Collaboration) Nucl. Phys. B 871, 1 (2013).

[17] D. Muller for the LHCb Collaboration, these proceedings.

[18] B.B. Abelev et al. (ALICE Coll.), Phys. Lett. B738, 97 (2014). 
[19] G. Aad et al. (ATLAS Collaboration), Phys. Rev. D 85, 052005 (2012).

[20] R. Baier et al., Nucl. Phys. B 483, 291 (1997).

[21] Y.L. Dokshitzer and D.E. Kharzeev, Phys. Lett. B 519, 199 (2001).

[22] M. Djordjevic, M. Gyulassy and S. Wicks, Phys. Rev. Lett. 94, 112301 (2005).

[23] J. Adam et al. (ALICE Collaboration), JHEP 1603, 081 (2016) and JHEP 09, 112 (2012).

[24] J. Adam et al. (ALICE Collaboration), JHEP 1603, 082 (2016).

[25] M. He, R.J. Fries and R. Rapp, Phys. Lett. B 735, 445 (2014).

[26] CMS Collaboration, CMS-HIN-16-001.

[27] J. Adam et al. (ALICE Collaboration), JHEP 11, 205 (2015).

[28] CMS Collaboration, CMS-PAS-HIN-12-014 and CMS-PAS-HIN-15-005.

[29] C.A. Salgado et al., J. Phys. G 39, 015010 (2012).

[30] J.W. Cronin, H.J. Frisch, M.J. Shochet, J.P. Boymond, P.A. Piroue and R.L. Sumner, Phys. Rev. D 11, 3105 (1975).

[31] A. Accardi, hep-ph/0212148.

[32] R.C. Hwa and C. Yang, Phys. Rev. Lett. 93, 082302 (2004).

[33] B. Abelev et al. (ALICE Coll.), submitted to JHEP (arXiv:1605.07569) and Phys. Rev. Lett. 113, 232301 (2014).

[34] K.J. Eskola, H. Paukkunen, C.A. Salgado, JHEP 0904, 65 (2009).

[35] H. Fujii and K. Watanabe, Nucl Phys. A 920, 78 (2013).

[36] CMS Collaboration, Phys. Rev. Lett. 116, 032301 (2016).

[37] CMS Collaboration, CMS-PAS-HIN-15-012.

[38] S. Moortgat for the CMS Collaboration, these proceedings.

[39] CMS Collaboration, Phys. Lett. B 754, 59 (2016).

[40] M. Nahrgang, J. Aichelin, P. B. Gossiaux and K. Werner, Phys. Rev. C 90, 024907 (2014).

[41] S. Cao, G.-Y. Qin, S. A. Bass and B. M§ller, J. Phys.: Conf. Ser. 446, 012035 (2013).

[42] J. Adam et al. (ALICE Collaboration), submitted to Eur. Phys. J. C (arXiv:1605.06963). 